\title{
BMJ Open Barriers to and discourses about breast cancer prevention among immigrant women in Spain: a qualitative study
}

\author{
Sebastià March, ${ }^{1}$ Barbara Villalonga ${ }^{2}$ Carmen Sanchez-Contador, ${ }^{3}$ Clara Vidal, ${ }^{1}$ \\ Aina Mascaro, ${ }^{4}$ Maria de Lluc Bennasar, ${ }^{1}$ Magdalena Esteva ${ }^{1}$
}

To cite: March S, Villalonga B, Sanchez-Contador C, et al. Barriers to and discourses about breast cancer prevention among immigrant women in Spain: a qualitative study. BMJ Open 2018;8:e021425. doi:10.1136/ bmjopen-2017-021425

- Prepublication history for this paper is available online. To view these files, please visit the journal online (http://dx.doi org/10.1136/bmjopen-2017021425).

Received 8 January 2018 Revised 4 October 2018 Accepted 5 October 2018

Check for updates

(C) Author(s) (or their employer(s)) 2018. Re-use permitted under CC BY-NC. No commercial re-use. See rights and permissions. Published by BMJ.

${ }^{1}$ Research Unit, Primary Care Department, Balearic Islands Health Service, Balearic Islands Health Research Institute (IdISBa), Palma, Spain

${ }^{2}$ Arquitecto Bennassar Health Center, Majorca Primary Care Department, Balearic Islands Health Service, Palma, Spain ${ }^{3}$ Balearic Department of Health, Breast Cancer Early Detection Program, Palma, Spain ${ }^{4}$ Majorca Primary Care Department, Balearic Islands Health Service, Palma, Spain

Correspondence to

Sebastià March;

sebastia.march@gmail.com

\section{ABSTRACT}

Objectives To identify knowledge, barriers and discourses about breast cancer screening in Spain among female immigrants from low-income countries and native Spanish women from a low socioeconomic class.

Design Qualitative interview study with thematic analysis interpreted using cultural mediators.

Setting Mallorca, Spain.

Participants Thirty-six in-depth interviews, using cultural mediators, of immigrant women living in Mallorca who were 50-69years old and were from Maghreb, SubSaharan Africa, Eastern Europe, Latin America, China or were native to Spain and from a low socioeconomic class. Results We analysed the interviews to assess breast cancer perceptions and beliefs, discourses about breast cancer prevention and barriers to accessing breast cancer prevention programmes. Although the women reported an association of breast cancer with death, they acknowledged the effectiveness of early detection. They also exhibited reluctance to talk about cancer. Discourses about cancer prevention tended to be proactive or fatalistic, depending on the woman's country of origin. For all women, fear of results and lack of time were barriers that limited participation in breast cancer prevention programmes. Language barriers, frequent changes of residence and fear due to status as an irregular (undocumented) immigrant were barriers specific to immigrant women.

Conclusions The culture of origin affects whether an immigrant has a fatalistic or proactive approach toward breast cancer screening. Immigrants from low-income countries and Spanish natives from a low socioeconomic class experience barriers in access to breast cancer screening. Frequently changing homes is also a barrier for immigrant women.

\section{INTRODUCTION}

Breast cancer is the most common cancer in Western women, excluding non-melanoma skin cancer. In 2012, there were an estimated 463000 incident cases and 131000 deaths from breast cancer in Europe. ${ }^{1}$ Early breast cancer detection is an effective measure for improving prognosis and reducing mortality, ${ }^{2}$ and many countries have therefore implemented screening programmes. ${ }^{3}$ However,

\section{Strengths and limitations of this study}

- The interviews of 36 female immigrants from low-income countries allowed examination of the effect of socioeconomic status and cultural origin on acceptance of breast cancer screening.

- Recruitment, interviews and analysis support were performed by female mediators mostly from the same geographic regions, using the languages of the respondents.

- A limitation is that women from some cultural and geographic areas were pooled together to simplify comparisons.

there are some controversies about the effectiveness of these programmes, and the balance between their harms and benefits. ${ }^{4}$ In Spain, women who are 50 to 69 years old and registered in the national census receive invitations for free biennial mammography. As of 2010, after our data collection was completed, these invitations included women who were irregular (undocumented) immigrants.

Several Spanish studies have shown that population-based screening reduces socioeconomic inequalities in access to mammography, in contrast to opportunistic screening, which is commonly used for cervical cancer. ${ }^{56}$ However, female immigrants from low-income countries are less likely to participate in cancer screening programmes than native Spanish women. ${ }^{78}$

About $11 \%$ of the total population of Spain consists of immigrants and culturally diverse individuals, and half of them are from low-income countries (Source: National Institute of Statistics). Between 2001 and 2007, 64\% of targeted women in the Balearic Islands accepted invitations to participate in the breast cancer prevention programme, but the acceptance rate was lower for women from less developed foreign countries (Maghreb: $44.9 \%$, Latin America: 43.2\%, Eastern Europe: 36\%, Sub-Saharan Africa: 26\%) 
(Source: Balearic breast cancer screening programme; data not published). The lower use of preventive medical procedures by immigrant women from low-income countries also occurs in other Western countries. ${ }^{9}{ }^{10}$ Some of the factors that reduce participation in programmes that screen for breast cancer are similar in immigrant and native women,${ }^{11}$ including old age, ${ }^{12}{ }^{13}$ low socioeconomic status, ${ }^{14}$ not having health insurance, ${ }^{15}$ not perceiving any risks or benefits, ${ }^{131516}$ being single ${ }^{12}$ and no recommendation for mammographic screening by a doctor. ${ }^{13}{ }^{14}$ However, immigrant women face the added difficulties of language barriers and cultural differences. Their culture defines their beliefs, values and rules, and these affect their attitudes towards cancer and cancer prevention. ${ }^{17-19}$ Moreover, the immigrant population in Spain is heterogeneous, and they have diverse beliefs and perceptions about breast cancer and its prevention. ${ }^{20} 21$ Some research suggests that socioeconomic status has a greater influence on discourse and willingness to participate in cancer screening than birthplace. ${ }^{22}$

Women from low-income countries have a lower incidence of breast cancer, but a worse prognosis following diagnosis of breast cancer than women from Western Europe. ${ }^{23}$ Therefore, to reduce the inequalities in access to prevention programmes and improve the prognosis in this population, we need to identify their perceptions of breast cancer screening, and the specific barriers they face for participation in screening.

The aims of our study were: to characterise the knowledge and perceptions of breast cancer and breast cancer screening of immigrant women from low-income countries who are living in Spain; to identify differences in the discourses about cancer prevention among immigrants from different geographical origins and native Spanish women with similar socioeconomic status; and to identify the barriers that prevent immigrant women from participating in breast cancer screening programmes. Our results will allow us to determine the relevance of socioeconomic status and culture in the country of origin on the willingness of immigrant women to participate in breast cancer prevention programmes and their dialogues about breast cancer.

\section{METHODS}

This qualitative interview study was performed in 20092010. The target population was immigrant women from low-income countries and native Spanish women who had low socioeconomic status, who were 50 to 69 years old and living in Majorca, Spain. To develop the theoretical sampling, we selected five groups of women from different geographic regions, with these groups accounting for the largest number of immigrant women: Maghreb (Morocco), Sub-Saharan Africa (Senegal, Mali), Eastern Europe (Romania, Bulgaria), Latin America (Colombia, Ecuador) and Asia (China). Immigrants from these five groups of countries account for more than $70 \%$ of all immigrants, and immigrants from Ecuador and
Colombia account for about $30 \%$ of Latin America immigrants. We randomly selected a woman of the selected nationalities from the Breast Cancer Programme database, and selected additional women using snowball sampling. Spanish women who were from boroughs with low socioeconomic status were randomly selected from the same database for comparisons. Within each group, interviews were conducted until the thematic saturation was reached, that is, when the last interview did not bring anything new according to the two analysts, and their results could be predicted. Intercultural women mediators were also selected to perform field studies of the immigrant women; five of these women were professional mediators who worked for the health system, and two were informal mediators identified by these professionals.

\section{Data collection}

Information was collected using in-depth, audio-recorded interviews by the intercultural mediators, using each immigrant's mother tongue and an original script previously developed by the research team. Native Spanish women were interviewed by female members of the research. Five mediators were also interviewed by members of the research team, as they are considered key agents in their communities.

All interviewers received a 6-hour training workshop. Mediators contacted the immigrant women by telephone, and explained the objectives of the study and scheduled an appointment for the interview. The women chose the place for the interview, in the primary health centre or at home, which lasted 30 to $90 \mathrm{~min}$. Before the interview, additional information on the study objectives were provided, and informed consent was obtained before recording. After the interview, each participant received a small gift.

The interview examined concepts of health and prevention, knowledge of cancer and screening programmes and previous experiences with mammography. The script was adapted during fieldwork to improve clarity and facilitate the work of the interviewers. Each interviewer led the interview, and did not perform a second interview until the transcript was reviewed jointly with members of the research team. Interviewers translated and transcribed the audio-recorded interviews following a protocol developed ad hoc by the research team, which promoted the taking of notes during the interview to provide a better understanding of cultural expressions or references. The interviews were in Arabic, Mandarin, Wolof, French, Romanian or Bulgarian, and were translated into Spanish before analysis.

\section{Sample description}

A total of 31 women plus five intercultural mediators were interviewed (table 1). The reasons for immigration were economic in most cases, except for women of Chinese and Moroccan origin, who mostly immigrated for family reunification. The native Spanish women were employed, had medium-to-low socioeconomic and educational 
Table 1 Characteristics of women in the study

\begin{tabular}{|c|c|c|c|c|c|c|}
\hline Origin & $\begin{array}{l}\text { Number of } \\
\text { interviews }\end{array}$ & Level of education & Years in Spain & $\begin{array}{l}\text { Reasons } \\
\text { for } \\
\text { migration }\end{array}$ & $\begin{array}{l}\text { Understanding } \\
\text { Spanish }\end{array}$ & $\begin{array}{l}\text { Previous } \\
\text { mammography }\end{array}$ \\
\hline Morocco & $\begin{array}{l}5 \\
+1 \mathrm{IM}\end{array}$ & Low & $5-10$ & FR & With difficulties & Yes \\
\hline China & 4 & Low & $<5$ & FR & No & No \\
\hline $\begin{array}{l}\text { Sub-Saharan } \\
\text { Africa }\end{array}$ & $\begin{array}{l}4 \text { Senegal } \\
1 \text { Mali } \\
+1 \mathrm{IM}\end{array}$ & Secondary & $5-10$ & Work & $\begin{array}{l}\text { Yes, with } \\
\text { difficulties }\end{array}$ & Variable \\
\hline Latin America & $\begin{array}{l}2 \text { Colombia } \\
3 \text { Ecuador } \\
+1 \mathrm{IM}\end{array}$ & Low/Secondary & $>10$ & Work & Yes & Yes \\
\hline Eastern Europe & $\begin{array}{l}5 \text { Bulgaria } \\
3 \text { Romania } \\
+2 \text { IM }\end{array}$ & University/Secondary & $5-10$ & FR/work & Yes & Yes \\
\hline
\end{tabular}

*Migration within Spain.

FR, family reunification; IM, intercultural mediator.

status, had urban backgrounds and moved to Majorca from other regions of Spain.

\section{Analysis}

Two researchers (SM and BV) independently performed a thematic analysis, ${ }^{24}$ based on preset codes regarding the objectives of the study, the information obtained from the field work and bibliographical review. During the analysis, new codes were developed that were incorporated into the analysis. Some of these new codes were related to the study objectives, but others were related to immigrant condition, gender identity and cultural issues (such as acceptance or resistance to Western medicine). The data from these additional codes were a useful complement that aided the interpretation of results.

First, each group was analysed, then a comparative analysis of the different groups was performed. A sociological analysis of the discourses ${ }^{25}$ regarding cancer prevention and perceived barriers to mammography was also performed. The position of the analysts was that beliefs and concepts related to health were influenced by their cultural origin at birth, but also by their social position (gender and social class).

To determine if there were sufficient interviews, preanalysis was used to detect saturation during fieldwork. Researchers and interviewers discussed the final results, and the latter's contributions were integrated into the study results.

\section{Patients and public involvement}

Participants were not involved in the development of the research questions. Cultural mediators, as representatives of the different cultures, participated in the development and review of the interview script, and results analysis. Results were returned to them and their contribution to results interpretation was incorporated into the definitive analysis.

\section{RESULTS}

\section{Cancer perceptions and beliefs}

All of the interviewed women reported associating cancer with death, the lack of an effective treatment and being an incurable disease; paradoxically, most of them knew that early detection increased the probability of cure. The mere mention of the word 'cancer' often evoked fear and dread, and many women did not want to discuss the topic. Moroccan women avoid the word 'cancer', and when it is mentioned, they automatically invoke divine protection.

(MOROCCO) Interviewer: Let's talk about another topic: cancer

Respondent: May God protect us and keep us away from that disease!

Sub-Saharan African and Chinese women also avoided talking about cancer. Chinese women associate talking about cancer with the probability of developing it. They quote a Chinese expression that says 'Anything going out of your mouth can come in again'.

Eastern European women shun discussion about cancer because of certain shame if they or their relatives suffer from it. Thus, to be polite, they avoid asking anyone about cancer.

(BULGARIA) Respondent: It may be due to religion, culture, education or shame, but you don't dare to tell anyone what is happening to you, sometimes you even don't dare to tell your mother what is happening to you.

This reluctance to talk about cancer means that most women are not interested in receiving information about cancer, including native Spanish women. 
(MOROCCO) Respondent: Better not, because the more you know the more it affects you and makes you ill. I don't like going into this subject.

Generally, the women just wanted to be informed when they actually have to face the problem of cancer, although women who have lived longer in Spain gave a higher value learning more about cancer. Being close to people with cancer, or having experienced cancer, seems to arouse interest in the subject.

\section{Discourses about prevention}

Two opposite discourses regarding health and disease among the interviewed women were identified, and these could impact acceptance of cancer prevention programmes. First, there was a fatalistic discourse about disease, in which an individual feels that 'what will happen to us is imposed by destiny'. For Moroccan women, this fatalistic discourse was expressed as the will of God. For native Spaniards, it was less well defined; they also used expressions that refer to God's will, but their underlying discourse did not seem religious. In contrast to the fatalistic discourse, some women had a proactive discourse. The proactive discourse focused on the relationship of health problems with individual lifestyles, in that each individual has his or her own responsibility regarding health outcomes. Fatalistic discourses were used to explain avoidance of disease prevention practices, as these individuals believed that 'whatever will be, will be'; women with more proactive discourses were more accepting of the need for disease prevention programmes. Although both discourses were present in women from all groups, Latin American women were more prone to proactive discourses, and native Spaniards to fatalistic discourses.

(SPAIN) Respondent: I prefer not know. If it has to come because God wants it and I have to run, ok. But to be worried when you are calm. Don't think so.

(MOROCCO) Respondent: If it is written that you will suffer from this disease, then you will have it, despite your measures, and if it is written that you will have a long life, then you will have it. This is why it is very difficult to associate it with prevention.

Religious beliefs did not necessarily lead to fatalism. For example, in the case of women from the Maghreb, Islam could have different effects on the discourse: it may lead to a fatalistic discourse or a proactive discourse, because the Koran calls for good Muslims to maintain healthy lifestyles. The relationship of a proactive discourse with religion was also present in women from Latin American countries.

(ECUADOR) Respondent: I am very Catholic and I always say: God said, take care of yourself and I will take care of you, but one has to take care of oneself.

Having a proactive discourse was related with more favourable attitudes towards cancer prevention programmes in Latin American and Moroccan women who requested and received mammography. Chinese and Eastern European women were more likely to express that they had no need for preventive testing, and could wait to seek medical care until symptoms were present. They claimed that these visits are very expensive in their own countries, and are only performed when absolutely necessary.

(CHINA) Respondent: Here in Spain, between 50 and 70 years of age all women must have an examination. It may be because here you don't have to pay for going to the doctor. We have to pay. If you discover a lump you go to the doctor, if you are all right you don't go.

Breast self-examination as a means of early detection is common among African, Chinese and especially Eastern European women. This practice is well known in these groups, because of its promotion by health authorities in these countries.

\section{Barriers to preventive testing}

All women had some knowledge of mammography, even those who never had one. Our interviews revealed several factors that could negatively affect acceptance of screening mammography. In particular, social position (gender and socioeconomic class) had a negative impact on acceptance of mammography in all groups, including native Spaniards, but other barriers seemed specific to immigrants (figure 1).

One of the main barriers for receiving screening mammography was a lack of time, especially when the reason for immigration was related to work. The immigrants reported that keeping their jobs was a high priority. Some of them had jobs with strict schedules and there were concerns about job security; this meant they may have been reluctant to take a leave from work. The native Spanish women also mentioned this as the main barrier to receiving screening mammography.

Moreover, women who immigrated looking for a job and had family members who depended on them said they feared that if a mammography detected cancer, this would force them to stop working. Women who immigrated for family reunification, such as those from China and Morocco, also declared having problems scheduling a screening mammography because of their responsibilities at home, such as child care, and their limited social support.

(BULGARIA) Respondent: It's simple. Immigrant women have just an objective: Working to help their families. and they know that they can easily lose their job, as another woman will do the same job and sometimes for less money.

(ROMANIA) Respondent: I once had the chance of doing some tests while I was working as a maid in a hotel, and my fellow worker told me: Hey! What if we are found to have some disease and get sacked? 


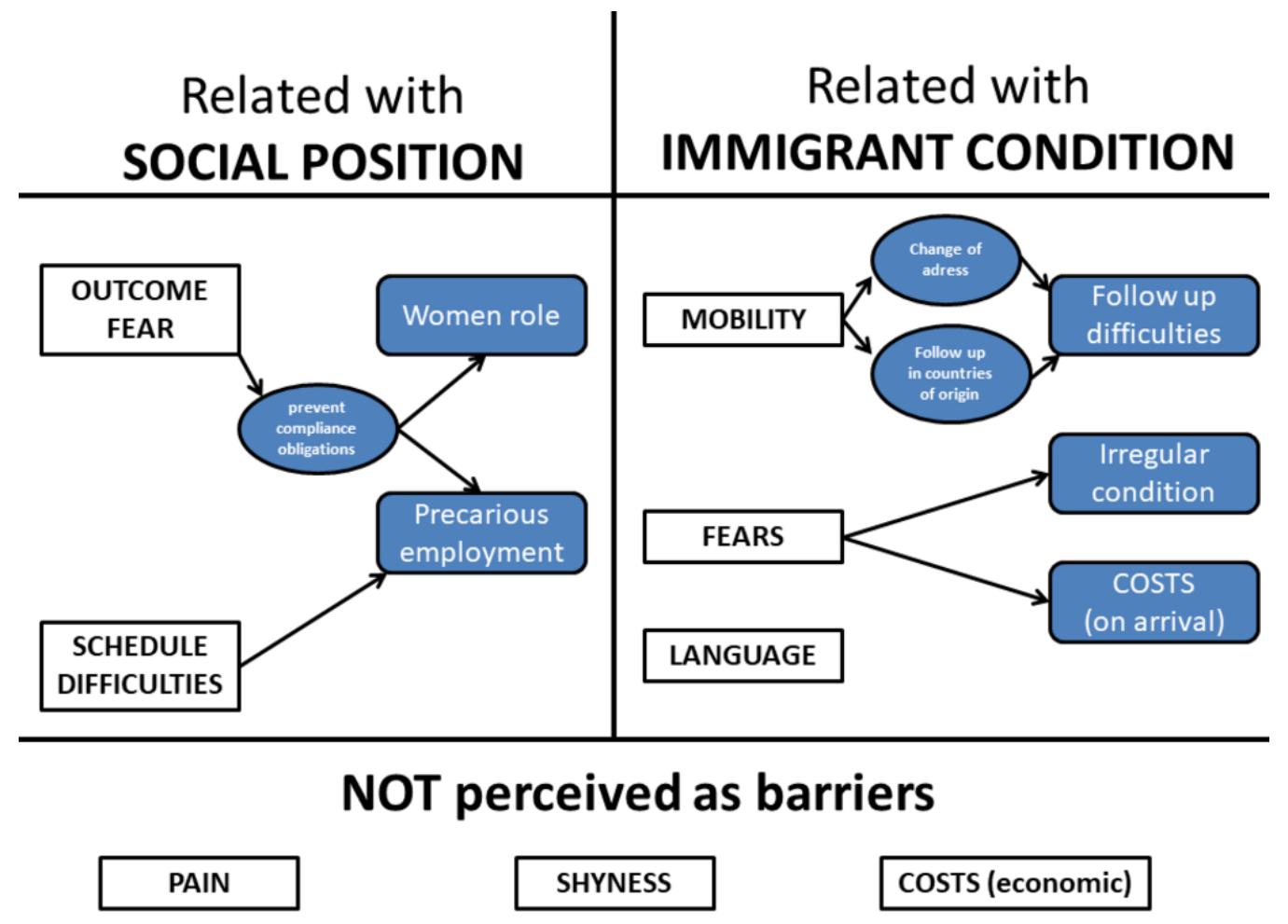

Figure 1 Barriers to participation in the population-based breast cancer screening programme.

Some unique aspects of the immigrant condition prevented women from accessing preventive services, mainly during their first years in Spain. These individuals frequently change homes, and often move to a new city or even a new region. This could make it difficult to keep their healthcare data updated in the Health System database, and prevent receipt of an invitation letter for screening mammography.

(SUB-SAHARAN AFRICA) Respondent: I didn't get any letter or it was maybe sent to the house where I used to live before travelling to Senegal.

Some immigrant women had preventive check-ups in their own countries. Because these women need to get their test results and other information from their countries of origin, this can be a barrier to maintaining proper follow-up in Spain. These women were mainly Chinese and Moroccan, probably because they had the greatest language barriers; but it was also true of some Latin American and Eastern European women, who preferred to receive screening in their home countries.

Moreover, some of the immigrant women were irregular (undocumented) immigrants. This may cause them to fear authorities and be reluctant to contact public health services, often because they are ignorant of their rights in Spain. When these women first arrived in Spain, many did not seek health services because they thought it would cost money or that they could be reported to the police. The letters that invite women to participate in the breast cancer screening programme do not clearly mention that screening mammography is free, as this is well understood in Spain. Thus, some women, such as those from Eastern Europe, had concerns about the cost of mammography. After women have lived in Spain for some time, this misunderstanding is no longer an issue.

(ROMANIA) Mediator: They do not know about available health resources, as well as health rights. There will be always the concern, 'And if I have to pay after?'

Language was the main barrier mentioned by some women, mainly those from Maghreb, China and Sub-Saharan Africa. Respondents from Eastern Europe claimed that after a short adaptation period, language was no longer a problem, although some Bulgarians still had difficulties reading, due to differences between the Cyrillic and Latin alphabets. Language barriers forced the women to depend on others, and this increased their feelings of insecurity. These women often had to be accompanied by a relative, and this compromised the confidentiality of medical visits. Chinese women sometimes paid a translator from the community, so they did not have to bother working relatives. Women from Maghreb and China mentioned that contact with a cultural mediator in hospitals and health centres reduced the impact of this barrier.

Religious and cultural differences did not seem to be insurmountable barriers for any of the interviewed women. Women from cultures with strong taboos about nudity, such as Morocco and China, prefer to be attended by a woman during mammography. However, none of the interviewed women claimed this was especially relevant, 
because they understood that being treated by a man is common in the Spanish health services.

Although many women acknowledged suffering some pain during mammography, none believed this was a barrier, because they considered the pain as bearable, and acceptable because of the benefits provided by the test. Latin American and Eastern European women mentioned the pain caused by mammography, although they claimed it would not prevent them from receiving one. Some Eastern European women said they were afraid of X-rays, and this made them reticent to receive mammography, because they believed the radiation applied during mammography was harmful.

(ROMANIA) Respondent: If you compare the experience of a mammography with what you have to live through when you have a cancer, it is like a mosquito bite. an insignificant pain.

Economic barriers were not an issue, except for recent immigrants who were not aware that mammographic screening was free. On the contrary, the immigrant women reported that the lack of free screening in their own countries was one of the main barriers to accessing preventive services.

\section{DISCUSSION}

Our results show that some of the barriers that limit access to breast cancer screening were related to gender and socioeconomic class, and were therefore present in immigrants and Spanish natives. However, other barriers were specific to immigrants. Also, the discourses of these women about cancer prevention were variable, and ranged from being proactive to fatalistic.

Most of the interviewed women showed a lack of interest in disease prevention in general, and cancer prevention in particular. This may be because all the interviewed women had low or medium-to-low socioeconomic status, and they had common barriers related to their fear that a diagnosis of cancer would prevent them from working. Reluctance to talk about cancer was common for women from China, Maghreb and Eastern Europe, because these women had different health beliefs about cancer. ${ }^{26}$ This lack of interest in prevention and screening, added to an inadequate communication about cancer, can lead to misconceptions, as previously described. ${ }^{27}$ Therefore, the resistance of these women to talk about cancer should be considered when planning interventions that seek to dismantle the misconceptions of these women and improve their health literacy.

We classified the women's discourses on prevention (and the conception of health) on an axis. On the one hand, some women had fatalistic discourses, in which they believed that their health is a matter of fate or luck, and beyond their own control. ${ }^{28}$ This discourse could lead to the underuse of preventive services. On the other hand, other women had more proactive discourses, in which they believed they could control and determine their own health by modifying their lifestyle. Parts of both discourses could be present in the same group, and even in the same woman. In this sense, although most of the interviewed women reported an association of cancer with death (fatalism), they also recognised the benefits of early detection (proactive). We also found that a relevant factor in the discourses was a woman's acceptance of Western medicine. Thus, some women had discourses of clear resistance (no confidence), but others had discourses that extolled the benefits of Western medical practices (confidence) (figure 2).

On one hand, we found proactive discourses, together with a high confidence in Western medicine, in Latin American women, and these women were more likely to participate in preventive programmes. On the other hand, we found fatalistic discourses and low confidence in the Western medicine, ${ }^{1628}$ in women from Eastern European, Sub-Saharan African and native Spanish women from a low socioeconomic class. Fatalistic discourses were more common in the native Spanish women. ${ }^{28}{ }^{29}$ Although we did not expect to find this discourse in Spanish women, our results are in line with those of other studies of individuals in low socioeconomic classes in other countries. ${ }^{30}$ Maghreb women were in an intermediate position, and their discourses seemed to be affected by their acculturation. ${ }^{31}{ }^{32}$ Higher integration (being settled in the country, having a job and speaking the language) seems to be related to having a more proactive discourse. Chinese respondents had a proactive discourse and attitude, but they distrusted Western medicine, and this may explain their low participation in preventive programmes. ${ }^{171833}$ These results are similar to those of other studies in Spain. ${ }^{81}$

The lack of time, due to responsibilities at home or in the workplace, was one of the most frequently mentioned barriers for participation in preventive mammography. This was associated with the low socioeconomic status of the women, as they tended to have insecure and low-skill jobs, making it difficult to take time off. The female gender was also a barrier, because women have specific roles in patriarchal cultures, such as caring for children, cooking and housekeeping. These problems were common in the immigrant and native women due to their similar socioeconomic status. Therefore, some of the barriers in accessing disease prevention programmes are due to socioeconomic class and gender, but immigrants face additional specific barriers. ${ }^{34}$ This result is in accordance with the results of other studies, ${ }^{11} 162035$ although other studies often emphasised psychological, sociocultural, organisational and structural factors. Our purpose was to identify the common and unique barriers of native Spaniards and immigrant women, and to determine which barriers are associated with low socioeconomic status and which are associated with status as an immigrant.

We found that fear of pain from screening mammography, resistance to being cared for by a male doctor and economic costs of mammography were not major barriers 


\section{PROACTIVE}

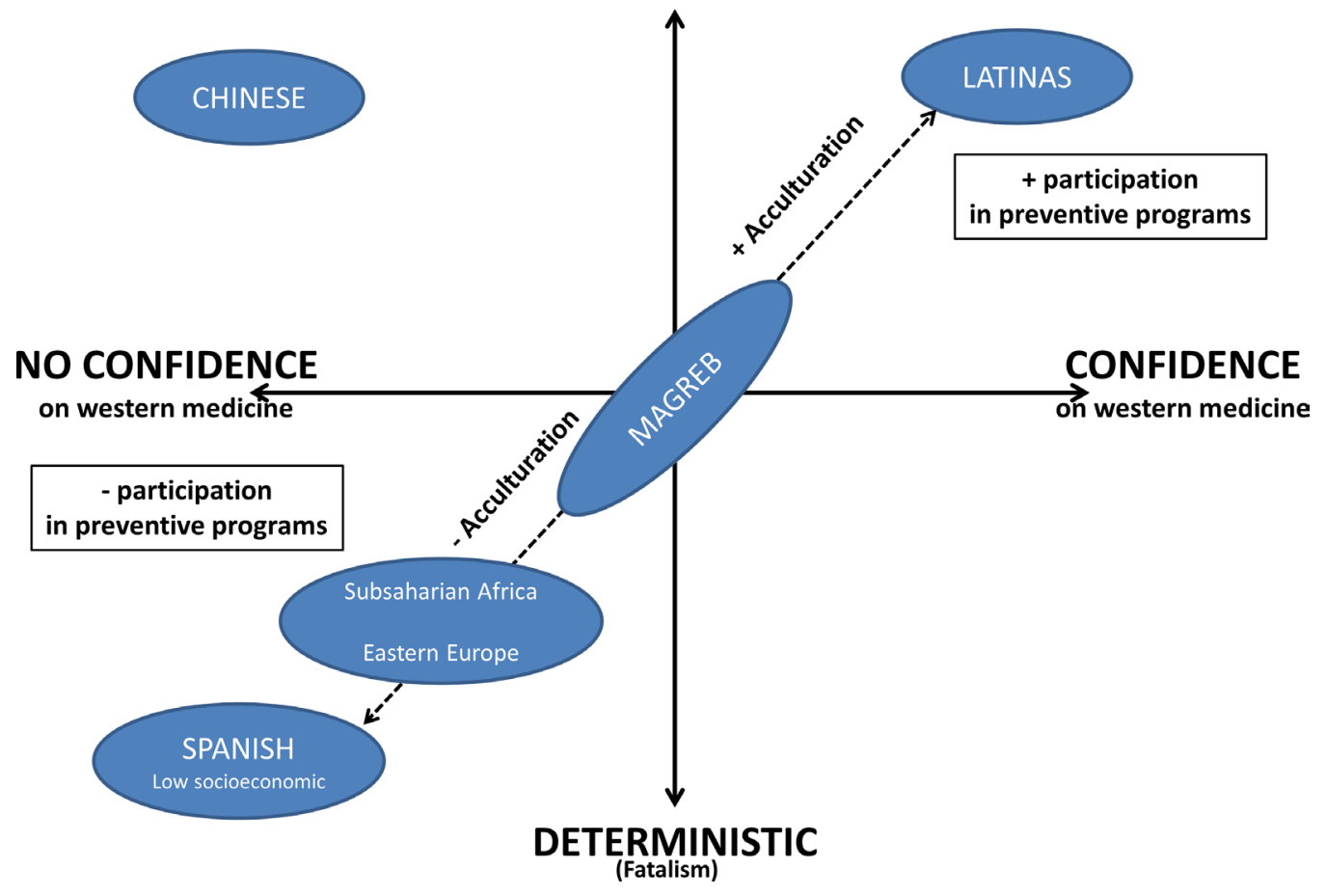

Figure 2 Characterisation of discourses as proactive or fatalistic, and confidence in the Western medicine.

for undergoing screening mammography, although these were identified as barriers in other studies. ${ }^{11} 1620$ It could be that the women downplayed the importance of first two reasons (pain from mammography and attended by a male doctor) to satisfy the expectations of the interviewer. On the other hand, it seems that the third reason (cost) was not an issue due to the universal access to public healthcare in Spain.

In 2012, after our data collection ended, Spain enacted a new law (RD16/2012) that limits the rights of irregular (undocumented) immigrants to access the public healthcare system. Therefore, many of the women who we examined will now face an additional barrier to accessing disease prevention services, and this could have a significant negative impact on their health and well-being. The authors of this article firmly oppose this measure, which is contrary to providing equal access to healthcare for all, the coexistence of immigrants with natives in Spain and protection of public health.

Our results indicate the existence of healthcare inequalities in Spain, in that immigrants have more limited access to breast cancer screening, and presumably to other disease prevention programmes. Measures are therefore needed to provide preventive services that guarantee equality of access to healthcare in Spain. At the health services level, these should include the development of messages tailored to the needs and discourses of immigrant groups; the incorporation of intercultural mediators into prevention programmes (because patient navigation programmes are not yet well developed in Spain); and periodic assessments of the participation of women with different origins and socioeconomic classes in prevention programmes, with a focus on equality. At the structural level, these measures should include the promotion of integration and regulations that facilitate the traceability of immigrants, ${ }^{8}$ so they can be reminded of the importance of disease prevention, and the development of social policies and advocacy that improve access to healthcare and protect the labour rights of immigrants and others in the lower socioeconomic classes. $^{36}$

\section{Strengths and limitations}

The main strength of this study is that we assured the validity of the results by use of several strategies. In particular, two researchers independently analysed all results and then discussed them with the intercultural mediators. None of the participants had a previous abnormal mammography. An abnormal result could have changed the perception of cancer and the discourses on cancer prevention of these women.

Although analysis of the discourses of women from five different regions was useful, it should be interpreted with caution at the group level, because it is an oversimplification of a very heterogeneous women population, made only for sampling and analysis purposes. We partially addressed this limitation by encouraging the participation of intercultural mediators for interpretation of the results. Another limitation is that Mallorca is not a representative region of Spain, because it has a very high percentage of immigrants. Even so, our results are consistent with those obtained in other regions of Spain. ${ }^{20} 21$ 


\section{CONCLUSIONS}

The discourses that women have about breast cancer screening and the factors that hinder their access to screening were similar for immigrants from low-income countries and Spanish natives from a low socioeconomic class. Therefore, cultural origin and immigration statusin addition to social position (gender and socioeconomic class)_influence participation in a breast cancer prevention programme and perceptions of cancer.

The interviewed women showed little interest in learning more about cancer, had difficulties scheduling appointments for mammographic screening and expressed fear that positive mammography results would limit their ability to continue to fulfil their traditional responsibilities that are imposed on them by their low socioeconomic class and gender. Immigrants had more barriers than native Spaniards, such as language difficulties, frequent changes in residence and fear about interacting with institutions if they were irregular (undocumented).

Acknowledgements We appreciate the collaboration of Elena Gonzalez in designing the study and training the interviewers. We also thank all of the intercultural mediators who developed the fieldwork: Soufane Oulkadi, Ndeye Bineta Diagne, Leida Pilar Cardenas, Narcisa Luzuriaga, Tania Todova Stoyanka, Monica Moldovan and Núria Villena. We are especially grateful to Francisco Campoamor for his delicate revision of the last draft of the manuscript.

Contributors SM, BV, CS-C, CV, AM, MdLB and ME developed the study design and shared responsibilities for training the interviewers and performing the field work. SM and BV performed the data analysis. SM was the coordinator of the study, and wrote the first draft of the manuscript. All researchers discussed the results, reviewed the manuscript and approved the final version.

Funding This study was financed with a grant from the Ministry of Health, Carlos III Institute (ISCIII), PI08/90628. It also received the support of the Health Promotion and Preventive Activities-Primary Health Care Network (redlAPP), sustained by the Ministry of Health, ISCIII and cofinanced by ERDF funds (RETIC RD06/0018, RD12/0005/0011, RD16/0007). This project was also supported by the Balearic Islands Health Research Institute (IIISBa).

Disclaimer The sources of public and competitive funding did not influence the design or results of the study.

Competing interests None declared.

Patient consent Obtained.

Ethics approval This study obtained the approval of the Majorca Primary Care Research Committee.

Provenance and peer review Not commissioned; externally peer reviewed.

Data sharing statement Data are available upon request to the corresponding author SM through email.

Open access This is an open access article distributed in accordance with the Creative Commons Attribution Non Commercial (CC BY-NC 4.0) license, which permits others to distribute, remix, adapt, build upon this work non-commercially, and license their derivative works on different terms, provided the original work is properly cited, appropriate credit is given, any changes made indicated, and the use is non-commercial. See: http://creativecommons.org/licenses/by-nc/4.0/.

\section{REFERENCES}

1. Ferlay J, Steliarova-Foucher E, Lortet-Tieulent J, et al. Cancer incidence and mortality patterns in Europe: estimates for 40 countries in 2012. Eur J Cancer 2013;49:1374-403.

2. Who. International agency for research on cancer. handbook for cancer prevention, breast cancer screening. 7th edn: IARC Press, 2002.

3. Ballard-Barbash R, Klabunde C, Paci E, et al. Breast cancer screening in 21 countries: delivery of services, notification of results and outcomes ascertainment. Eur J Cancer Prev 1999;8:417-26.
4. Independent UK Panel on Breast Cancer Screening. The benefits and harms of breast cancer screening: an independent review. The Lancet 2012;380:1778-86.

5. Cabeza E, Esteva M, Pujol A, et al. Social disparities in breast and cervical cancer preventive practices. Eur J Cancer Prev 2007;16:372-9.

6. Puigpinós-Riera R, Serral G, Pons-Vigués M, et al. Evolution of inequalities in breast and cervical cancer screening in Barcelona: population surveys 1992, 2001, and 2006. J Womens Health 2011;20:1721-7.

7. Sanz-Barbero B, Regidor E, Galindo S. Influencia del lugar de origen en la utilización de pruebas de cribado de cáncer ginecológico en España. Revista de Saúde Pública 2011;45:1019-26.

8. Pons-Vigués M, Puigpinós-Riera R, Rodríguez-Sanz M, et al. Preventive control of breast and cervical cancer in immigrant and native women in Spain: the role of country of origin and social class. Int J Health Serv 2011;41:483-99.

9. Lagerlund M, Maxwell AE, Bastani R, et al. Sociodemographic predictors of non-attendance at invitational mammography screening-a population-based register study (Sweden). Cancer Causes Control 2002;13:73-82.

10. Vermeer B, Van den Muijsenbergh ME. The attendance of migrant women at the national breast cancer screening in the Netherlands 1997-2008. Eur J Cancer Prev 2010;19:195-8.

11. George SA. Barriers to breast cancer screening: an integrative review. Health Care Women Int 2000;21:53-65.

12. Edwards $\mathrm{NI}$, Jones DA. Uptake of breast cancer screening in older women. Age Ageing 2000;29:131-5.

13. Esteva M, Ripoll J, Leiva A, et al. Determinants of non attendance to mammography program in a region with high voluntary health insurance coverage. BMC Public Health 2008;8:387.

14. Shields M, Wilkins K. An update on mammography use in Canada. Health Rep 2009;20:7-19.

15. Champion VL, Skinner CS. Differences in perceptions of risk, benefits, and barriers by stage of mammography adoption. $J$ Womens Health 2003;12:277-86.

16. Remennick $L$. The challenge of early breast cancer detection among immigrant and minority women in multicultural societies. Breast $J$ 2006;12:S103-S110.

17. Lee-Lin F, Menon U, Nail L, et al. Findings from focus groups indicating what Chinese American immigrant women think about breast cancer and breast cancer screening. J Obstet Gynecol Neonatal Nurs 2012;41:627-37.

18. Kwok C, White K. Cultural and linguistic isolation: the breast cancer experience of Chinese-Australian women - a qualitative study. Contemp Nurse 2011;39:185-94.

19. Watts T, Merrell J, Murphy F, et al. Breast health information needs of women from minority ethnic groups. J Adv Nurs 2004;47:526-35.

20. Pons-Vigués M, Puigpinós-Riera R, Serral G, et al. Knowledge, attitude and perceptions of breast cancer screening among native and immigrant women in Barcelona, Spain. Psychooncology 2012;21:618-29.

21. Pons-Vigués M, Puigpinós-Riera R, Rodríguez D, et al. Country of origin and prevention of breast cancer: beliefs, knowledge and barriers. Health Place 2012;18:1270-81.

22. Hsia J, Kemper E, Kiefe C, et al. The importance of health insurance as a determinant of cancer screening: evidence from the women's health initiative. Prev Med 2000;31:261-70.

23. Harper S, Lynch J, Meersman SC, et al. Trends in areasocioeconomic and race-ethnic disparities in breast cancer incidence, stage at diagnosis, screening, mortality, and survival among women ages 50 years and over (1987-2005). Cancer Epidemiology Biomarkers \& Prevention 2009;18:121-31.

24. Aronson J. A pragmatic view of thematic analysis. The Qualitative Report 1995;2:1-3.

25. Ruiz J. Sociological discourse analysis: methods and logic. Forum Qual Soc Res 2009;10

26. Thomas VN, Saleem T, Abraham R. Barriers to effective uptake of cancer screening among Black and minority ethnic groups. Int $J$ Palliat Nurs 2005;11:562-71.

27. Chamot E, Perneger TV. Misconceptions about efficacy of mammography screening: a public health dilemma. J Epidemiol Community Health 2001;55:799-803.

28. Straughan PT. Fatalism reconceptualized: a concept to predict health screening behavior. Journal of Gender, Culture, and Health 1998;3:85-100.

29. Vrinten C, Wardle J, Marlow LA. Cancer fear and fatalism among ethnic minority women in the United Kingdom. Br J Cancer 2016:114:597-604 
30. Keeley B, Wright L, Condit CM. Functions of health fatalism: fatalistic talk as face saving, uncertainty management, stress relief and sense making. Sociol Health IIIn 2009;31:734-47.

31. Facione NC, Katapodi M. Culture as an influence on breast cancer screening and early detection. Semin Oncol Nurs 2000;16:238-47.

32. Page JB. The concept of culture: a core issue in health disparities. $J$ Urban Health 2005;82:iii35-iii43.

33. Joseph G, Burke NJ, Tuason N, et al. Perceived susceptibility to illness and perceived benefits of preventive care: an exploration of behavioral theory constructs in a transcultural context. Health Educ Behav 2009;36:71S-90.
34. Schoueri-Mychasiw N, Campbell S, Mai V. Increasing screening mammography among immigrant and minority women in Canada: a review of past interventions. J Immigr Minor Health 2013;15:149-58

35. Schueler KM, Chu PW, Smith-Bindman R. Factors associated with mammography utilization: a systematic quantitative review of the literature. J Womens Health 2008;17:1477-98.

36. Pons-Vigués M, Diez È, Morrison J, et al. Social and health policies or interventions to tackle health inequalities in European cities: a scoping review. BMC Public Health 2014;14:198. 